\title{
Emociones que emergen durante el análisis del conocimiento didáctico del contenido sobre el campo eléctrico
}

\author{
Emotions emerging during the analysis \\ of pedagogical content knowledge on electric field
}

Lina Melo ${ }^{1}$. Florentina Cañada ${ }^{2}$

\begin{abstract}
Resumen: El dominio afectivo integrado con la historia personal y social de los profesores, sus creencias y representaciones cognitivas, configura el gran espectro de conocimiento didáctico del contenido (CDC). El estudio que presentamos analiza las emociones declaradas de forma explícita, por dos profesores de bachillerato, dentro de un estudio de carácter cualitativo sobre el desarrollo del CDC en el caso de la enseñanza del campo eléctrico. La presencia de emociones es un indicador de las coincidencias o discrepancias entre los distintos componentes de su CDC e influyen en las decisiones que el profesor toma en el aula. Los contenidos más conflictivos, en términos emocionales, resultan ser la enseñanza de la superposición de campos y fuerzas eléctricas y la relación electrostática-electrocinética. Finalmente, las causas tanto de las emociones positivas como de las negativas están mayoritariamente relacionadas con el conocimiento curricular y el contenido que se enseña.
\end{abstract}

Palabras claves: Emociones. Contenido didáctico del conocimiento. Enseñanza de la física. Campo eléctrico.

\begin{abstract}
This study deals with the affective domain integrated with the personal and social history of teachers, and their beliefs and cognitive representations. This defines the shape of pedagogical content knowledge (PCK) when teachers teach and plan a concept. Our study analyzes the emotions declared by two high school teachers in a qualitative study on the development of pedagogical content knowledge in the case of teaching the electric field. The array of emotions is an indicator of coincidences or discrepancies between PCK components, and the influence of decisions taken by the teacher. The most conflictive aspects of content in emotional terms are overlapping electric fields, and forces and the electro-kinetic relationship. Finally, the causes of both, positive and negative, are related to curriculum and content knowledge.
\end{abstract}

Keywords: Emotions. Educational content knowledge. Physics teaching. Electric field.

\footnotetext{
${ }^{1}$ Universidad de Extremadura, Facultad de Formación del Profesorado, Departamento de Didáctica de las Ciencias Experimentales y de las Matemáticas, Cáceres, España. Orcid: < http://orcid.org/0000-0003-4771-058X>.

E-mail: <lvmelo@unex.es>.

${ }^{2}$ Universidad de Extremadura, Facultad de Educación, Departamento de Didáctica de las Ciencias Experimentales y de las Matemáticas, Badajoz, España. Orcid: < http://orcid.org/0000-0001-5544-0423>.
} 


\section{Introducción}

A partir de los estudios de Shulman (1986) se han desarrollado de manera fecunda diversas investigaciones referentes a la naturaleza, características e implicaciones del Conocimiento Didáctico del Contenido (CDC) en el conocimiento profesional de los profesores de ciencias. La mayoría de las investigaciones, con un fundamento constructivista, han mostrado que, aunque los conocimientos académicos, en ciencias, pedagogía y didáctica son esenciales en la formación y el desarrollo profesional no son suficientes, se requiere de escenarios donde el profesor reconozca y desarrolle su CDC sobre temas concretos de su enseñanza.

La agenda actual en didáctica de la física ha posicionado al conocimiento didáctico del contenido como un marco teórico vigente en la formación del profesorado, a pesar de distinciones epistemológicas de los conocimientos-base implicados: conocimiento de la disciplina, conocimiento sicopedagógico, conocimiento en didáctica de la física y del contexto.

Los resultados reportados sobre los aprendizajes disciplinares adquiridos por los profesores, sobre los contenidos de física que enseñan y su repercusión en el aprendizaje, son un aliciente que ratifica la necesidad de este tipo de conocimiento, en un panorama donde son cada vez más los profesionales de otras áreas los que se dedican a la enseñanza de la física.

Para todos los casos, la relación más estudiada ha sido aquella en la que se piensa la transformación, asociación, o transferencia del conocimiento disciplinar al conocimiento didáctico del contenido, situación que subyace a la concepción misma de la didáctica como disciplina y la tradición heredada de Shulman (1986). Aunque se ha privilegiado esta ruta, cada vez son más las investigaciones (GARRITZ, 2010; GESS-NEWSOME, 2015; MELLADO et al., 2014; SHULMAN, 2015; VAN DRIEL; BERRY; MEIRINK, 2014; ZEMBYLAS, 2007, 2011) que reclaman la presencia del dominio afectivo dentro de la enseñanza y la formación del profesorado.

Mellado et al. (2014) y Shulman (2015) indica que los aspectos afectivos sobre la comprensión de la enseñanza y la práctica de la enseñanza son importantes porque mucho de lo que el profesor conoce y hace está conectado a sus propios estados emocionales y motivaciones, e influye en el aprendizaje de sus estudiantes.

Garritz (2010) plantea la necesidad de reconocer el dominio afectivo, expresado en: emociones hacia el contenido que se enseña, la enseñanza y el aprendizaje, actitudes, y eficacia docente; como una componente más para garantizar su inclusión dentro de los análisis del CDC. Otros autores (GESS-NEWSOME, 2015; MELLADO et al., 2014; VAN DRIEL; BERRY; MEIRINK, 2014) la proponen como la lente catalizadora de la cognición y la acción del profesorado, la cual cumple diferentes funciones: fuente del CDC, movilizador del conocimiento de la disciplina, parte del conocimiento psicopedagógico y didáctico, y como elemento que configura las orientaciones sobre la enseñanza de las ciencias.

El objetivo de nuestro trabajo es caracterizar las emociones, que emergen del proceso de análisis y caracterización del CDC durante dos años consecutivos, antes y después de la participación de los profesores en procesos de intervención e innovación sobre la enseñanza del campo eléctrico. Finalmente, asumimos que el dominio afectivo, al igual que los conocimientos base que configuran el CDC, se transforma e integra en los procesos de innovación y desarrollo profesional sobre contenidos específicos. 


\section{Metodología}

La investigación se desarrolló con dos profesores de física colombianos de bachillerato, a quienes nos referiremos como Isabel y Alejandro. Isabel, con edad de 28 años y experiencia docente de cinco años en secundaria y dos en bachillerato, trabaja en una institución educativa de carácter femenino y privado. Alejandro, con edad de 30 años y experiencias docente de 7 años en la enseñanza de la física en bachillerato y un año en la formación del profesorado de primaria, trabaja en un colegio masculino de carácter privado. La edad de los estudiantes se encuentra entre los 17 y 19 años, y los grupos son de 15 a 30 estudiantes. Las dos instituciones educativas son de nivel socioeconómico alto, ubicadas en la ciudad de Bogotá.

Los instrumentos utilizados durante esta investigación incluyen: (a) un cuestionario de preguntas abiertas sobre lo que el profesor considera que son las estrategias de enseñanza en física y el papel de la planificación en el proceso de enseñanza-aprendizaje; (b) la plantilla dispuesta por Pro (1998) para realizar planificaciones; (c) la matriz diseñada por Loughran, Mulhall, y Berry (2004), como representación del contenido (ReCo); (d) entrevista semiestructurada, antes y después de las observaciones de clase; (e) los diarios de campo; y, (f) los registros etnográficos correspondientes a las sesiones del proceso de intervención.

La intervención se diseñó a partir del diagnóstico inicial del CDC. Se les propuso a los profesores plantear y evaluar una nueva unidad didáctica para la enseñanza del campo eléctrico en la cual se integrara la mayor cantidad de elementos propios de las reflexiones producto de la intervención. La decisión de los aspectos trabajados se consensuaron con los profesores según sus intereses desde tres fuentes: (a) las componentes del CDC; (b) los intereses y dificultades de los profesores hacia la enseñanza del campo y la fuerza eléctrica; y, (c) las emociones experimentadas durante la enseñanza del campo eléctrico. Las sesiones de la intervención se distribuyeron en reuniones formativas donde se trabajaron, situaciones estáticas que requerían del campo eléctrico, estrategias de enseñanza, herramientas de evaluación, dificultades de aprendizaje y discusión de propuestas innovadoras sobre la enseñanza de la electrostática, reuniones de reflexión sobre el CDC desde lo que los profesores dicen, diseñan y hacen y reuniones para el diseño y evaluación de la nueva unidad didáctica. Asumimos que los procesos de intervención con el profesorado deben basarse en la reflexión continua, autorregulada y metacognitiva, a partir de su contexto de trabajo cotidiano sobre contenidos específicos, a través de procesos de construcción de conocimiento compartido (MELO; CAÑADA; DÍAZ, 2017).

Somos conscientes que los instrumentos utilizados, tal como señalan Sutton, Knight y Mudrey-Camino (2009), limitan la caracterización de la mirada multidimensional de la emoción, porque permite profundizar en la experiencia subjetiva y las tendencias de acción de la emoción, pero no en los cambios fisiológicos.

La sistematización de los datos y su análisis se realiza siguiendo las técnicas de análisis de contenido (BARDIN, 1986) que incluyen los siguientes pasos (SOLÍS; PORLÁN; RIVERO, 2012): (a) identificación de las unidades de información procedentes de cada instrumento; (b) codificación de las unidades de información en las categorías y subcategorías; (c) categorización de las unidades de información; (d) análisis de las unidades de información; (e) incorporación de categorías emergentes. Siempre recurriendo al contenido manifiesto, con el fin de mantener los parámetros de fiabilidad y verificabilidad. 
Para la identificación de las unidades de información realizamos sucesivas lecturas de todos los instrumentos, tanto para el primer año de investigación como para el segundo, a través del software de análisis de datos cualitativos Nvivo 10. Los resultados fueron presentados a tres investigadores expertos para confirmar la relevancia y fiabilidad de la descripción.

Para la categorización, asumimos una visión multicomponencial de la emoción planteada por Sutton y Wheatley (2003), donde el proceso emocional consta de una red de cambios en una variedad de subsistemas (o componentes) del organismo. Estos componentes incluyen usualmente la evaluación, la experiencia subjetiva, el cambio fisiológico, la expresión emocional, y las tendencias a la acción. Estos componentes se interrelaciones, pero son independientes. Para nuestra investigación sólo analizamos las descripciones explícitas que los profesores realizan, sobre sus tendencias de acción, y las valoraciones de las situaciones vividas. En el Cuadro 1 presentamos el sistema de categorías final (MELO; CAÑADA; MELLADO, 2017).

Como se muestra en la tabla 1, las fuentes que vinculan las expresiones emocionales son: los contenidos, el diseño curricular, la relación con sus estudiantes y la relación con el contexto. En todos los casos se realizó una diferenciación entre emociones positivas y negativas. La clasificación entre emociones positivas y negativas se realizó siguiendo los parámetros establecidos en investigaciones anteriores, con profesores en formación de primaria (BRÍGIDO et al., 2013) y secundaria (BORRACHERO; COSTILLO; MELO-NIÑO, 2013), donde se consideran las emociones negativas: miedo, ira, tristeza, asco, ansiedad y vergüenza; y emociones positivas: alegría, amor y felicidad.

\section{Resultados}

A través del análisis de contenido identificamos aquellas frases que hacen alusión a emociones positivas y negativas, señaladas de forma explícita por los profesores, mientras razonan sobre las distintas decisiones que toman sobre la enseñanza del campo eléctrico. A continuación describiremos las emociones reportadas por Isabel y Alejandro.

\section{Emociones declaradas por Isabel}

En el Gráfica 1 se resumen las emociones que la profesora declara hacia la enseñanza de los contenidos e ideas concretas que enumera durante la enseñanza del campo eléctrico. Un total de 134 unidades de información clasificadas en el 2011, y 152 unidades en el 2012.

Las emociones declaradas, hacia la enseñanza de la carga, campo y fuerza eléctrica (F2.1), durante el año 2011 son altamente positivas en un 68\% frente al 32\% de las negativas. El mayor número de emociones positivas están vinculadas a la enseñanza de las líneas de campo y la solución de ejercicios sobre la fuerza eléctrica, y un mayor número de emociones negativas relacionadas con la superposición de las fuerzas eléctricas. La capacidad y confianza, en lo que se hace y dice, son los elementos más considerados en sus respuestas durante el primer año, seguidos de la preocupación y ansiedad. Esta imagen no está lejos de su idea de aprendizaje del campo eléctrico, donde la visualización del campo es considerado el elemento fundamental para su enseñanza. 
Cuadro 1. Categorías utilizadas para caracterizar las Emociones

\begin{tabular}{|c|c|c|c|c|}
\hline & Contenido & Diseño Curricular & $\begin{array}{c}\text { Relación con los } \\
\text { Estudiantes }\end{array}$ & $\begin{array}{l}\text { Relación con el } \\
\text { Contexto }\end{array}$ \\
\hline $\begin{array}{l}\quad \quad \mathbf{F 1} \\
\text { Emociones } \\
\text { hacia la } \\
\text { enseñanza } \\
\text { de la física }\end{array}$ & $\begin{array}{l}\quad \text { F1.1 } \\
\text { Recoge las } \\
\text { experiencias } \\
\text { o creencias } \\
\text { relacionadas } \\
\text { hacia los } \\
\text { contenidos de } \\
\text { física, desde } \\
\text { sus creencias, la } \\
\text { planificación y } \\
\text { su acción. }\end{array}$ & \begin{tabular}{l}
\multicolumn{1}{c}{ F1.2 } \\
Da cuenta de \\
situaciones \\
relacionadas con \\
la evaluación, el \\
patrón temático, \\
la metodología \\
y estrategias de \\
enseñanza de la \\
física.
\end{tabular} & \begin{tabular}{l}
\multicolumn{1}{c}{ F1.3 } \\
Fundamentada en \\
la relación frente a \\
la participación de \\
los estudiantes y sus \\
actitudes frente a la \\
física y las actividades \\
propuestas.
\end{tabular} & \begin{tabular}{l}
\multicolumn{1}{c}{ F1.4 } \\
Parte de los \\
requerimientos \\
institucionales y de \\
la coherencia entre \\
las concepciones \\
del profesor y la \\
propuesta educativa \\
institucional. Además \\
de las situaciones \\
relacionadas con los \\
padres de familia y los \\
compañeros de trabajo.
\end{tabular} \\
\hline $\begin{array}{l}\quad \text { F2 } \\
\text { Emociones } \\
\text { hacia la } \\
\text { enseñanza } \\
\text { del Campo } \\
\text { Eléctrico }\end{array}$ & \begin{tabular}{l}
\multicolumn{1}{c}{ F2.1 } \\
Recoge las \\
experiencias \\
relacionadas \\
hacia el campo, \\
fuerza y carga \\
eléctrica, desde \\
sus creencias, la \\
planificación y \\
su acción.
\end{tabular} & \begin{tabular}{l}
\multicolumn{1}{c}{$\mathbf{F} 2.2$} \\
Da cuenta de \\
situaciones \\
relacionadas con \\
la evaluación, el \\
patrón temático, \\
la metodología \\
y estrategias \\
de enseñanza \\
relacionadas con \\
la carga, fuerza y \\
campo eléctrico.
\end{tabular} & $\begin{array}{l}\text { F2.3 } \\
\text { Respuesta a las } \\
\text { situaciones, actitudes } \\
\text { y emociones de las } \\
\text { estudiantes frente a la } \\
\text { implementación del } \\
\text { diseño curricular y la } \\
\text { unidad didáctica para } \\
\text { la enseñanza de la } \\
\text { electrostática. También } \\
\text { sobre la gestión del aula } \\
\text { y la relación con sus } \\
\text { estudiantes. }\end{array}$ & \begin{tabular}{l}
\multicolumn{1}{c}{ F2.4 } \\
Da cuenta de \\
situaciones relacionadas \\
los las propuestas \\
del profesor sobre \\
la enseñanza del \\
campo eléctrico y su \\
repercusión con la \\
propuesta educativa \\
institucional.
\end{tabular} \\
\hline
\end{tabular}

Fuente: elaborado por las autoras.

Gráfica 1. Emociones declaradas sobre la enseñanza de contenidos concretos por Isabel

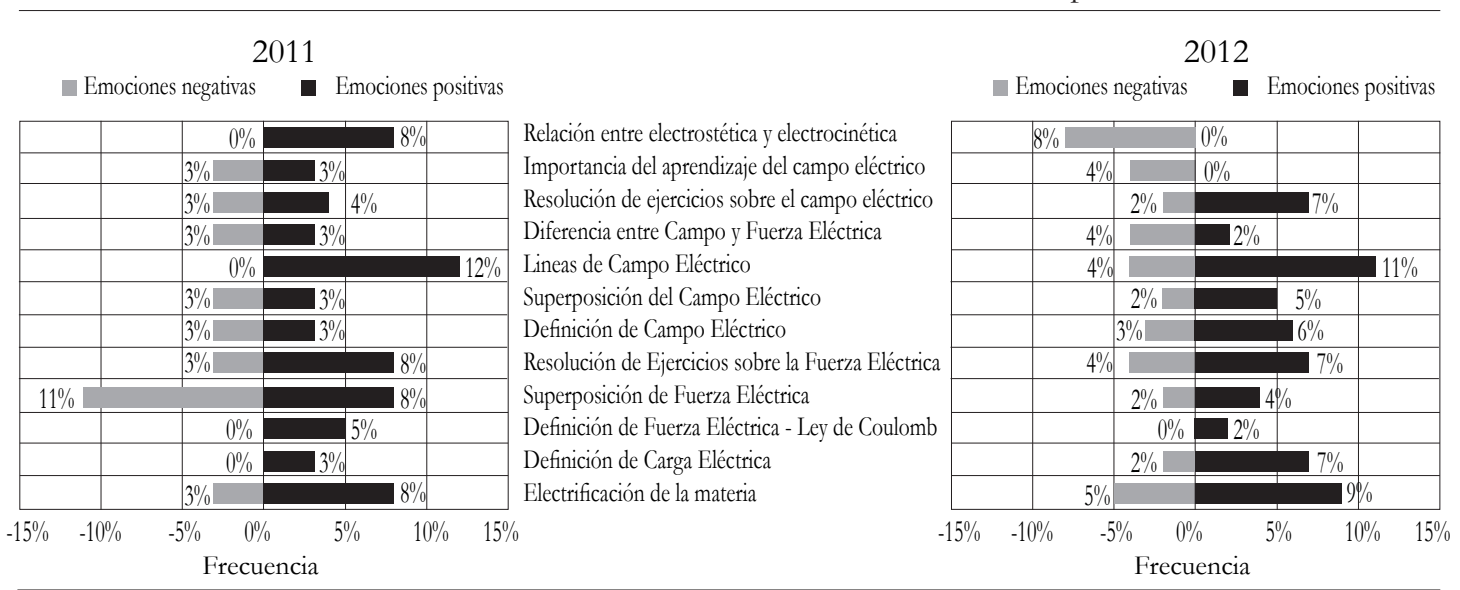

Fuente: elaborado por las autoras. 
Los resultados de las emociones negativas se deben a la falta de estrategias por parte de la profesora a la hora de enseñar los vectores y la idea de campo, un contenido que encuentra muy complejo para sus estudiantes, una toma de conciencia por parte de la profesora sobre su falta de dominio del contenido que está enseñando y las propias dificultades que la profesora ha experimentado durante su proceso de formación escolar y universitaria hacia la matemática y el aprendizaje del campo eléctrico. También influye la creencia sobre los estudiantes "nopueden entender la física porque no son buenos en matemáticas".

Durante el año 2012, un 60\% de las alusiones dan cuenta de emociones positivas frente a un $40 \%$ de las negativas. La profesora identifica un mayor número de emociones positivas vinculadas con la enseñanza de la carga eléctrica, el campo, las líneas de campo y la diferenciación entre fuerza y campo eléctrico, en comparación con el primer año. La capacidad, satisfacción y seguridad son las emociones más citadas.

La profesora señala que el proceso de intervención le ayudó a afianzar todas aquellas dudas que tenía sobre su conocimiento y el proceso de enseñanza elegido durante el primer año, pero a la vez, los cambios sobre la estructuración de la idea de campo eléctrico le generó tensión, ansiedad y preocupación. También es foco de emociones negativas el pensar llevar al aula la relación entre los circuitos y la electrostática, un aspecto sobre el que no había reflexionado el primer año, al igual que tener que justificar a los estudiantes la importancia de la idea de campo, cuando considera que no es necesaria en bachillerato.

Profundizando en las causas de las emociones, como se muestra en la Gráfica 2, a nivel de la enseñanza de la física encontramos que la mayor causa de emociones, tanto positivas como negativas, está referida al currículo (F1.2), seguidas de la relación con los estudiantes (F1.3) y el contexto (F1.4). Son pocas referencias las que realiza sobre los contenidos de física en general (F1.1), ya que constituye el fundamento de su elección laboral. Además agrega que dejaría la enseñanza si no se sintiera entusiasmado por su trabajo y los contenidos que enseña. Sin embargo expresa como sus experiencias universitarias hacia la matemática fueron muy negativas, situación que modela sus orientaciones hacia la relación de la matemática como herramienta para la física y media su enseñanza, porque que aquellos conceptos que más matemática vincula le son más difíciles de enseñar y mayor insatisfacción le generan. Al respecto menciona:

En la universidad la física me gustaba pero sentía siempre muchos vacios porque tenía que ver mucho con la matemática y mi matemática era pésima [...]. En medio de lo difícil que era para uno, admitir que uno no tenía el mismo nivel de los demás, se fue dando un, una, como un planteamiento y un reto personal, pero la pase muy mal [F1.1], y [...] pues los temas desde mi experiencia los que les cuestan [a los estudiantes] son los que más parte matemática tiene, porque a pesar de que tengan poca o mucha, no son tan fáciles de mostrar, por ejemplo, las leyes de Newton yo se los cuento en clase y ellas me dicen si es cierto, pero de abi a que detrás de calcular la aceleración de ese cuerpo yo tenga que coger el vector descomponerlo hacer toda una secuencia lógica, gráfica y matemática eso les hace que sea menos digerible para ellas y más,..., para mí, o sea... [F1.2] $]^{3}$

\footnotetext{
${ }^{3}$ Entrevista realizada entre los años de 2010-2011.
} 
En general, Isabel considera muy positivo, en términos emocionales, el apoyo y la relación con la institución educativa (F1.4). Respecto a la relación con los estudiantes se aprecia que el segundo año es más positiva que el primero (F1.3), fundamentalmente se debe al cambio de estudiantes de un año a otro y a la percepción global que tiene la profesora sobre el rendimiento académico de sus estudiantes.

Con relación a las emociones relacionadas con la enseñanza del campo eléctrico, Isabel declara como emociones positivas relacionadas con el currículo (F2.2): (i) confianza y satisfacción sobre la congruencia entre los objetivos de aprendizaje propuestos y los diseños planteados para una actividad experimental, con los resultados de aprendizaje obtenidos; (ii) alegría por apoyar sus clases con más experimentos que al inicio de su docencia; (iii) satisfacción sobre el diseño curricular porque cumple con sus expectativas hacia el aprendizaje; (iv) alegría hacia su enseñanza porque a medida que pasan los años aprovecha mejor el tiempo de la clase; (v) placer por enseñar porque es una oportunidad para aprender nuevas cosas, o comprende mejor otras. También son fuentes de emociones positivas el apoyo que la institución educativa le brinda en el desarrollo de su enseñanza, lo cual la dota de una mayor confianza para asumir los retos del día a día (F2.4).

Gráfica 2. Causas de las Emociones declaradas por Isabel

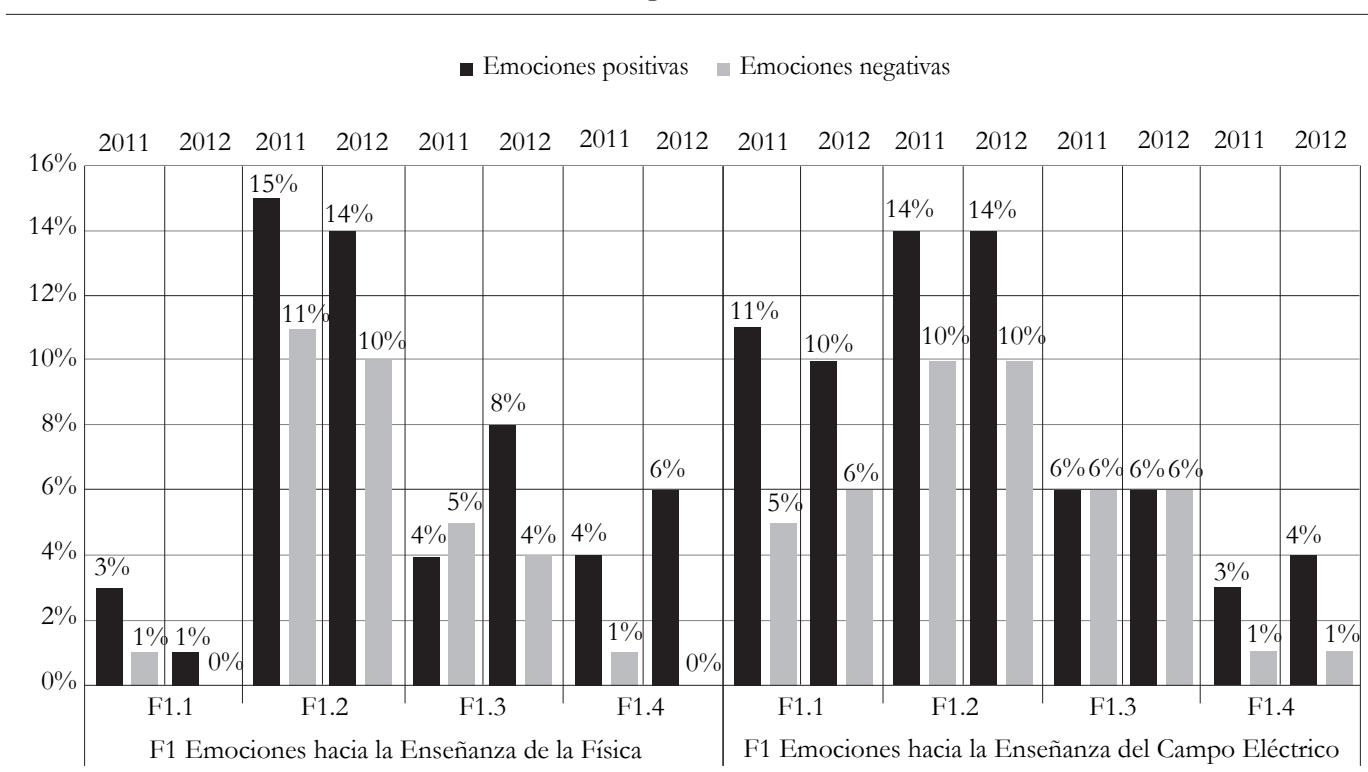

Fuente: elaborado por las autoras.

El análisis de las emociones negativas (ansiedad, preocupación, decepción, y frustración) sobre el currículo y la metodología (F2.2) se deben a la falta de tiempo para proponer acciones que cumplan su idea de un aprendizaje centrado en las estudiantes, su desconocimiento sobre 
estrategias específicas de enseñanza, y el posible cuestionamiento de su eficacia como profesor por las acciones y decisiones que ha tomado durante la enseñanza del campo eléctrico por su entorno social. También señala como contenido de emociones negativas la actitud de sus estudiantes durante las clases. Isabel considera que esa actitud descalifica su trabajo previo y el tiempo invertido en el diseño de sus clases (F2.3), lo cual hace que opte por una planificación mental y una enseñanza tradicional. Durante el segundo año, de nuevo los factores curriculares son los que más emociones positivas y negativas le provocan.

Durante toda la investigación, Isabel como profesora novel, en la enseñanza en bachillerato, al pensar en la profesión docente o como ella es como profesor tienen emociones mezcladas (F1.2, F1.3). Sin embargo muestra altos niveles de energía y entusiasmo, hacia la enseñanza de la física en general, y se ha comprometido con la propuesta de innovación pedagógica sobre la enseñanza del campo eléctrico (F2.2). Situación que difiere de los profesores en formación (LAMBSON, 2010) los cuales suelen sentirse confundidos, estresados e indefensos.

\section{Emociones declaradas por Alejandro}

En la Gráfica 3 resumimos las emociones que el profesor declara hacia la enseñanza de los contenidos concretos que menciona durante la enseñanza del campo eléctrico. Un total de 183 unidades de información clasificadas en el 2011 y 91 durante el 2012.

Gráfica 3. Emociones declaradas sobre la enseñanza de contenidos concretos por Alejandro

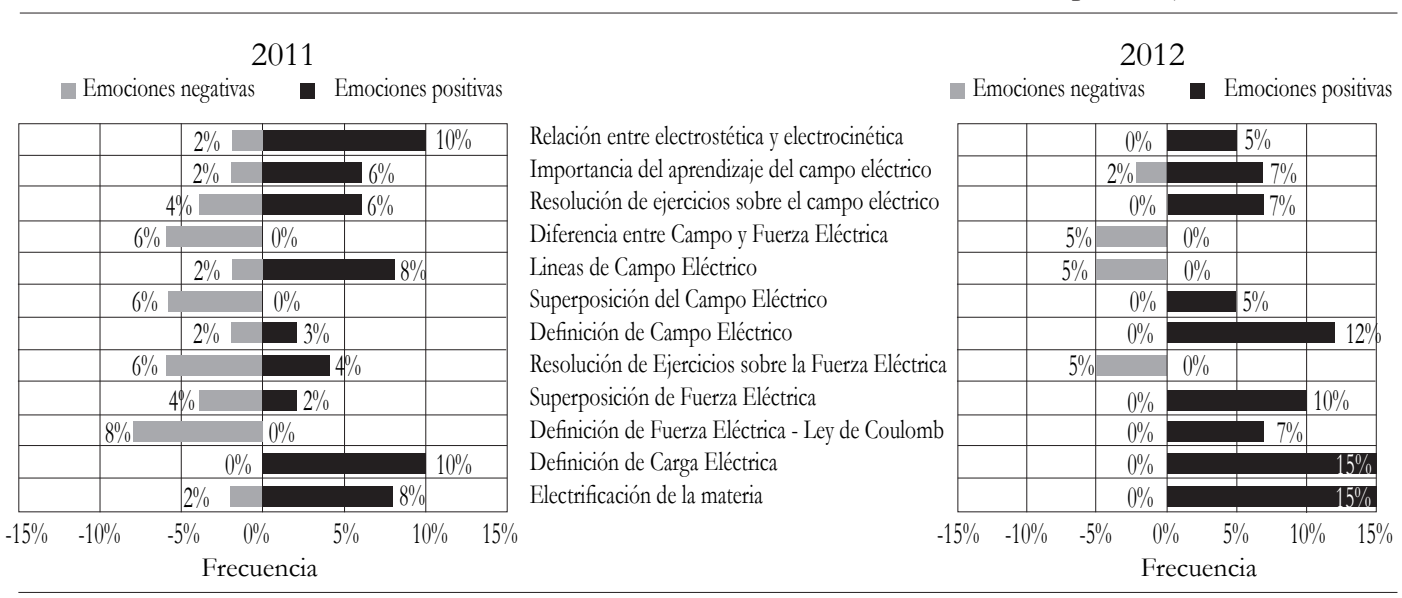

Fuente: elaborado por las autoras.

Las emociones declaradas hacia la enseñanza de la carga, campo y fuerza eléctrica durante el 2011, son altamente positivas en un 56\% frente al 44\% de las negativas (F2.1). El mayor número de emociones positivas está vinculado con la relación entre lo cinético y lo estático, aunque durante la descripción de su conocimiento curricular no caracterice dicha relación. La 
capacidad, fascinación y simpatía en lo que se hace y dice son los elementos más considerados en sus respuestas, seguidos de incertidumbre, pesimismo, decepción y aburrimiento, frente a los resultados de las evaluaciones y del aprendizaje en general.

Los resultados de las emociones negativas están relacionados con un descenso en la motivación de sus estudiantes, debido a sus propias estrategias centradas en el profesor. Sin embargo estas emociones no repercuten lo suficiente en la confianza que el profesor tiene sobre la eficacia de su enseñanza de tal forma que propicie un cambio. Otras emociones negativas hacen referencia a dificultades de aprendizaje que persisten en sus estudiantes después de la instrucción, como son la diferenciación entre fuerza y campo, o la superposición de campos.

Durante el 2012, y con la idea de mostrar la fuerza como efecto del campo, un 83\% de las alusiones dan cuenta de emociones positivas frente a un $17 \%$. El profesor identifica un mayor número de emociones positivas vinculadas con la enseñanza de la carga y fuerza eléctrica. La capacidad, satisfacción, confianza y simpatía son las emociones positivas más citadas, y decepción y frustración en las negativas. Esta última tiene que ver con la realización de la actividad de laboratorio de las líneas equipotenciales, y la confusión que declara entre éstas y las líneas de fuerza. En definitiva, optar por la secuencia de contenidos donde la fuerza es el efecto del campo antes de iniciar la enseñanza del campo eléctrico incrementa las emociones positivas hacia su enseñanza.

En comparación con Isabel, las emociones reportadas son en menor cuantía hacia la enseñanza de la física (F1) y el papel de la institución educativa en su proceso de enseñanza (F1.4); las negativas están relacionadas con la relación que mantiene sobre los estudiantes (F1.3) y el proceso de evaluación (F1.2). Al respecto comenta:

[...] el estudiante nunca va a ser sincero al ahora de responder una evaluación, y nunca va a ser capaz de decir, no sé, y va a preferir, tratar de engañarlo a uno que ser sincero, y me frustra, entonces eso a que lleva, a que uno como docente está jugando, o está planteando mal la evaluación, entonces la evaluación hay que..., debe resignificarse. [F1.2] $]^{4}$

Ahondando en los aspectos del contenido de las emociones hacia la enseñanza del campo eléctrico encontramos que la mayoría son referidas al currículo y la metodología, como representamos en la Gráfica 4.

Los resultados de las emociones positivas durante el primer año están relacionados con el valor que ha dado a la necesidad de mostrar una física que está relacionada con el mundo real y de utilidad (F1.1). Esta meta desde sus descripciones, no tiene valor cognitivo, solamente un valor afectivo, y su fin es incrementar la motivación y las actitudes positivas hacia la física, situación que le compensa emocionalmente, más cuando se trata de la enseñanza del campo eléctrico (F2.1).

Durante los dos años, al igual que Isabel, los factores curriculares son los que más emociones positivas y negativas suscitan hacia la enseñanza del campo eléctrico, especialmente los relacionados con la organización de los contenido, los resultados de las evaluaciones y la

\footnotetext{
${ }^{4}$ Entrevista realizada entre los años de 2010-2011.
} 
facilidad que el profesor considera que tiene para la creación de espacios de discusión entre los estudiantes, coherente con su idea de aprendizaje hacia la construcción de explicaciones, son también fuentes de emociones positivas (F2.2).

Las emociones negativas están relacionadas con el descenso en la motivación de sus estudiantes hacia el aprendizaje de la carga, campo y fuerza eléctrica, asumida como responsabilidad del estudiante. Durante el segundo año, el incremento de las emociones negativas se da por la inseguridad que expresa el profesor al plantear una nueva secuencia de contenidos para la enseñanza del campo eléctrico, donde la fuerza es el eje central de sus explicaciones y no la carga eléctrica. Otras emociones negativas hacen referencia a dificultades de aprendizaje que persisten en sus estudiantes después de la instrucción, debidas al nivel de abstracción del contenido, entre ellas considerar que campo y fuerza eléctrica son lo mismo, y la actitud que asumen sus estudiantes frente a dichas dificultades (F2.3).

Respecto al papel de la institución educativa, Alejandro considera que apoya sus iniciativas y da libertad para desarrollar procesos de innovación sobre la enseñanza de la física, siempre que mantenga los buenos resultados de las pruebas saber 11, situación que genera tanto emociones positivas como negativas (F1.4, F2.4). Específicamente durante el segundo año, no hace referencia a este aspecto a nivel declarativo.

Gráfica 4. Causas de las Emociones declaradas por Alejandro

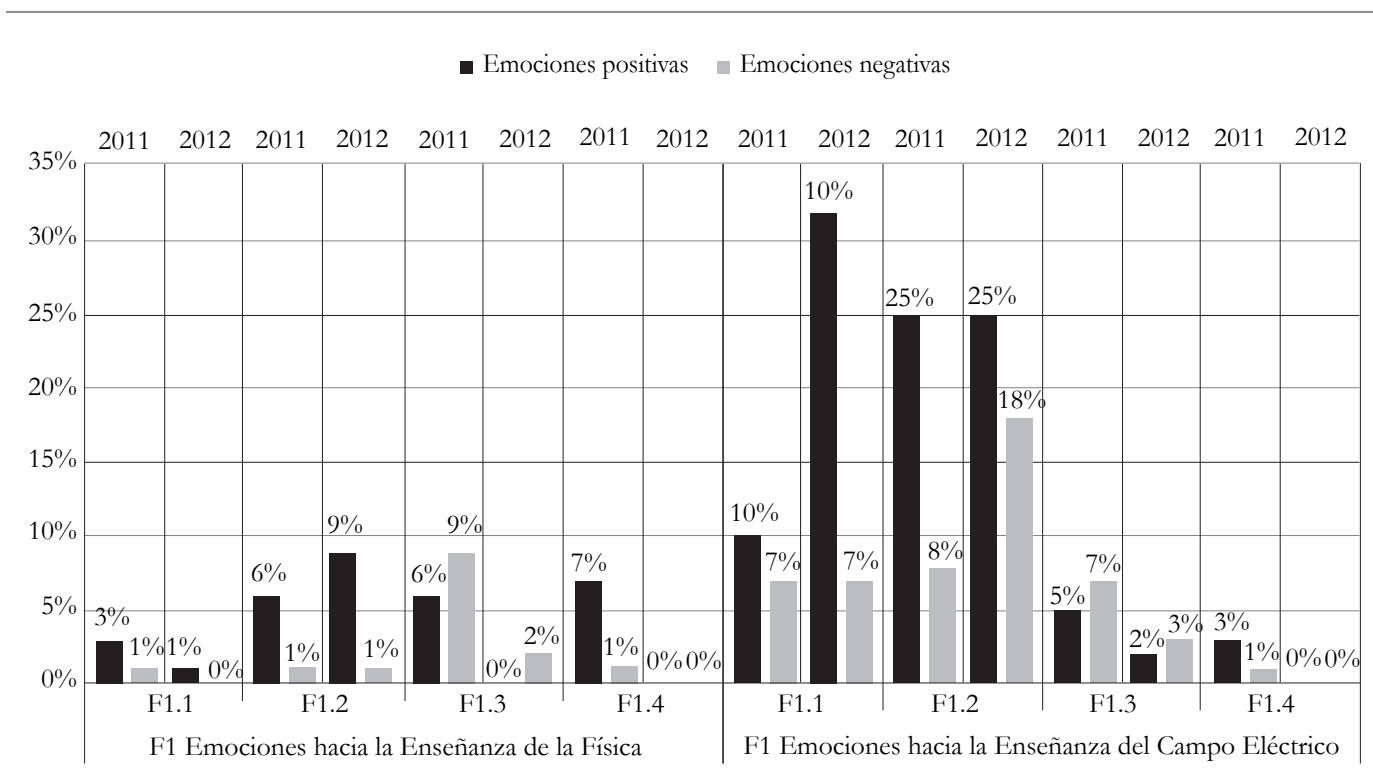

Fuente: elaborado por las autoras. 


\section{Conclusiones y Discusión}

Los dos aspectos fundamentales que emergen en el discurso sobre las emociones tienen relación con: (i) las fuentes del CDC, sus expectativas sobre el aprendizaje de los estudiantes y la relación con ellos; (ii) la proposición de estrategias particulares de enseñanza y los resultados de su diseño curricular; (iii) el contenido que enseña; y, (iv) la relación con la institución educativa, su contexto laboral, y sus compañeros de trabajo. También las creencias de autoeficacia hacia el contenido que se enseña, la evaluación y las estrategias de enseñanza resultan un mediador en el proceso E-A, y por supuesto la actitud de los profesores hacia la física, la enseñanza y el aprendizaje. Somos conscientes de que las emociones positivas y negativas que cada profesor describe no son similares, y dependen: del contexto de enseñanza, el contexto personal, y el uso que dan a las palabras para describir sus emociones (BISQUERRA, 2000).

Desde la caracterización realizada, las emociones influyen principalmente en el conocimiento sobre el contenido, los estudiantes, el currículo y las estrategias de enseñanza. Los contenidos más conflictivos en términos emocionales resultan ser la enseñanza de la superposición de campos y fuerzas eléctricas y la relación electrostática-electrocinética. Las causas, tanto de las positivas como las negativas, están mayoritariamente relacionadas con el conocimiento curricular y el contenido que se enseña.

El caso de Isabel, demuestra que, aunque durante el primer año, optó por prácticas tradicionales de enseñanza debido mayoritariamente a las emociones negativas que experimentó, el segundo año adquirió nuevos conocimientos profesionales gracias a la combinación de emociones positivas y negativas hacia el currículo, las estrategias de enseñanza y contenidos a enseñar, que catalizaron sus cambios. Alejandro sin embargo, mostró muchas emociones positivas hacia el contenido. Su alto grado de satisfacción y felicidad con lo que estaba haciendo, se convierten en obstáculos hacia el cambio de su CDC, y hacen que Alejandro continúe utilizando estrategias conservadoras. Sutton y Wheatley (2003) señalan al respecto, que cuando un profesor utiliza estrategias conservadoras se reduce la imprevisibilidad de la clase. Esto hace que disminuya la probabilidad de que ocurran eventos inesperados o fracasos y hace que el profesor experimente menos emociones negativas.

Apoyando nuestros resultados, Zembylas (2007) concluye que el conocimiento emocional es una parte importante del CDC. El estudio de caso que desarrolla con distintos profesores, muestra que conectan sus comprensiones emocionales con lo que saben del contenido, la pedagogía, los discursos que llevan al aula, el currículo y sus historias personales. Freitas, Jiménez y Mellado (2004), Mellado (1998) y Vázquez et al. (2012) señalan que los cambios estructurales y curriculares no bastan para provocar un cambio didáctico entre los profesores, si los cambios no contribuyen a darles satisfacción personal en el trabajo y les compensa afectivamente (BELL, 1998; PEME-ARANEGA et al., 2008). Es decir el dominio afectivo está mediando el conocimiento profesional, pero a su vez, está relacionado específicamente con el contenido que enseña el profesor.

Consideramos que el sistema de categorización sobre las emociones es un punto de inicio para futuras investigaciones sobre el CDC y su relación con el dominio afectivo, lo cual posibilita nuevas líneas de investigación. También lo son los resultados sobre las causas de las emociones positivas y negativas. En nuestro estudio las emociones negativas excesivas hacia la enseñanza del campo eléctrico, sin alternativas viables, pueden llevar al maestro a la parálisis, 
la frustración y el agotamiento. Las emociones positivas, por otro lado, pueden llevar al profesorado a una zona de confort que también puede obstaculizar su cambio didáctico. Por tanto, una combinación de emociones positivas y negativas hace que los profesores tengan más potencial para el cambio. Sin embargo se requiere de otros estudios sobre contenidos concretos, que vinculen de forma integral lo cognitivo y lo afectivo como parte del aprender a enseñar.

En concordancia con lo que señala Zembylas (2011) es necesario mirar con más cuidado las narraciones e historias del profesor, para ver cómo hay una amplia evidencia de cómo el conocimiento emocional forma parte del conocimiento práctico y personal del profesor. Pero no es suficiente hacer explícitas las emociones y el CDC para generar cambios didácticos, se requiere de la proposición de nuevos instrumentos que permitan caracterizar con mayor profundidad el papel de las emociones como filtros y amplificadores del desarrollo del CDC.

\section{Referencias}

BARDIN, L. E1 análisis de contenido. Madrid: Akal 1986.

BELL, B. Teacher development en science education. In: FRASER, B. J.; TOBIN, K. (Ed.). International handbook of science education. Dordrecht: Kluwer, 1998. p. 681-694.

BISQUERRA, R. Educación emocional y bienestar. Barcelona: Praxis, 2000.

BORRACHERO, A. B.; COSTILlO, E.; MELO-NIÑO, L. V. Diferencias en las emociones como estudiante y docente de asignaturas de ciencias de secundaria. In: MELLADO, V. et al. (Ed.). Las emociones en la enseñanza y el aprendizaje de las ciencias y las matemáticas. Badajoz: DEPROFE, 2013. p. 373-394.

BRÍGIDO, M. et al. The emotions about teaching and learning science: a study of prospective primary teachers in three Spanish universities. Journal of Baltic Science Education, Siauliai, v. 12, n. 3, p. 299-311, 2013. Disponible en: $<$ http:/ /www. scientiasocialis.lt/jbse/files/pdf/vol12/299-311.Brigido_JBSE_Vol.12.3.pdf > . Accedido el: 22 ene. 2018.

FREITAS, M. I.; JIMÉNEZ, R.; MELLADO, V. Solving physics problems: the conceptions and practice of an experienced teacher and an inexperienced teacher. Research in Science Education, Dordrecht, v. 34, n. 1, p. 113-133, 2004.

GARRITZ, A. Personal reflection: pedagogical content knowledge and the affective domain of scholarship of teaching and learning. International Journal for the Scholarship of Teaching and Learning, Statesboro, v. 4, n. 2, p. 1-6, 2010. Disponible en: <https://doi. org/10.20429/ijsotl.2010.040226>. Accedido el: 22 ene. 2018.

GESS-NEWSOME, J. A model of teacher professional knowledge and skill including PCK: results of the thinking from the PCK summit. In: BERRY, A.; FRIEDRICHSEN, P.; LOUGHRAN, J. (Ed.). Re-examining pedagogical content knowledge in science education. New York: Routledge, 2015. p. 28-42. 
LAMBSON, D. Novice teacher learning through participation in a teacher group. Teaching and Teacher Education, Kidlington, v. 26, n. 8, p. 1660-1668, 2010.

LOUGHRAN, J.; MULHALL, P.; BERRY, A. In search of pedagogical content knowledge in science: developing ways of articulating and documenting professional practice. Journal of Research in Science Teaching, Reston, v. 41, n. 4, p. 370-391, 2004.

MELLADO, V. Preservice teachers' classroom practice and their conceptions of the nature of science. In: FRASER, B. J.; TOBIN, K. (Ed.). International handbook of science education. Dordrecht: Kluwer, 1998. p. 1093-1110.

MELLADO, V. et al. Las emociones en la enseñanza de las ciencias. Enseñanza de las Ciencias, Barcelona, v. 32, n. 3, p. 11-36, 2014. Disponible en: <https://doi.org/10.5565/ rev/ensciencias.1478>. Accedido el: 22 ene. 2018.

MELO, L; CAÑADA, V.; DÍAZ, M. Formación continua del profesorado de física a través del conocimiento didáctico del contenido sobre el campo eléctrico en bachillerato: un caso de estudio. Caderno Brasileiro de Ensino de Física, Florianópolis, v. 34, n. 1, p. 131-151, 2017. Disponible en: < https:// doi.org/10.5007/2175-7941.2017v34n1p131>. Accedido el: 23 ene. 2018.

MELO, L; CAÑADA, V.; MELLADO, V. Exploring the emotions in pedagogical content knowledge about the electric field. International Journal of Science Education, Abingdon, v. 39, n. 8, p. 1025-1044, 2017. Disponible en: < https://doi.org/10.1080/095006 93.2017.1313467>. Accedido el: 23 ene. 2018.

PEME-ARANEGA, C. et al. El proceso de reflexión orientado como una estrategia de investigación y formación: estudio longitudinal de caso. TED: Tecné, Episteme y Didaxis, Bogotá, n. 24, p. 75-98, 2008. Disponible en: <https://doi.org/10.17227/01203916.394>. Accedido el: 23 ene. 2018.

PRO, A. Planificación de unidades didácticas por los profesores: análisis de tipos de actividades de enseñanza. Enseñanza de las Ciencias, Barcelona, n. 17, v. 3 , p. 411-429, 1998. Disponible en: < http:/ /www.raco.cat/index.php/Ensenanza/article/ view/21594/21428>. Accedido el: 23 ene. 2018.

SHULMAN, L. Those who understand: knowledge growth in teaching. Educational Researcher, Thousand Oaks, v. 15, n. 2, p. 4-14, 1986.

. PCK: its genesis and exodus. In: BERRY, A.; FRIEDRICHSEN, P.; LOUGHRAN, J. (Ed.). Re-examining pedagogical content knowledge in science education. New York: Routledge, 2015. p. 3-13.

SOLÍS, E.; PORLÁN, R.; RIVERO, A. ¿Cómo representar el conocimiento curricular de los profesores de ciencias y su evolución? Enseñanza de las Ciencias, Barcelona, v. 30, n. 3, p. 9-30, 2012. Disponible en: <http://www.raco.cat/index.php/Ensenanza/article/ view/285681/373653>. Accedido el: 23 ene. 2018. 
SUT'TON, R. E.; KNIGHT, C.; MUDREY-CAMINO, R. The relationship among teachers' emotions and classroom management. Theory into Practice, Philadelphia, v. 48, n. 2 , p. $130-137,2009$.

SUTTON, R. E.; WHEATLEY, K. Teachers' emotions and teaching: a review of the literature and directions for future research. Educational Psychology Review, New York, v. 15, p. 327-358, 2003.

VAN DRIEL, J.; BERRY, A.; MEIRINK, J. Research on science teacher knowledge. In: ABELL, S.; LEDERMAN, N. (Ed.). Handbook of research on science education. New York: Routledge, 2014. p. 848-870.

VÁZQUEZ, B. et al. The process of change in science teachers' professional development: a case study based on the types of problems in the classroom. Science Education, Hoboken, v. 96, n. 2 , p. 337-363, 2012.

ZEMBYLAS, M. Emotional ecology: the intersection of emotional knowledge and pedagogical content knowledge in teaching. Teaching and Teacher Education, Kidlington, v. 23 , n. 4, p. 355-367, 2007.

Teaching and teacher emotions: a post-structural perspective. In: DAY, C.; LEE,

J. C-K. (Ed.). New understanding on teacher's work: emotions and educational change.

Dordrecht: Springer, 2011. p. 31-44.

Artigo recebido em 11/05/2017. Aceito em 19/06/2017.

Dirección para contacto: Universidad de Extremadura, Facultad de Formación del Profesorado, Departamento de Didáctica de las

Ciencias Experimentales y de las Matemáticas, Av. de la Universidad, s/n, 10071 Cáceres, España. 